\title{
BIEMBEDDINGS OF STEINER TRIPLE SYSTEMS IN ORIENTABLE PSEUDOSURFACES WITH ONE PINCH POINT
}

\author{
A. D. FORBES, T. S. GRIGGS, C. PSOMAS and J. ŠIRÁŇ \\ Department of Mathematics and Statistics, The Open University, Walton Hall \\ Milton Keynes MK7 6AA, United Kingdom \\ e-mails: anthony.d.forbes@gmail.com,t.s.griggs@open.ac.uk, \\ c.psomas@gmail.com,j.siran@open.ac.uk
}

(Received 26 August 2012; accepted 24 January 2013; first published online 13 August 2013)

\begin{abstract}
We prove that for all $n \equiv 13$ or $37(\bmod 72)$, there exists a biembedding of a pair of Steiner triple systems of order $n$ in an orientable pseudosurface having precisely one regular pinch point of multiplicity 2 .
\end{abstract}

2010 AMS Classification. 05B07, 05C10.

1. Introduction. A complete graph $K_{n}$ has a triangulation in an orientable surface if and only if $n \equiv 0,3,4$ or $7(\bmod 12)$. The necessity of the condition follows immediately from Euler's formula $V+F-E=2-2 g$, where $V, F$ and $E$ are the numbers of vertices, faces and edges, respectively, and $g$ is the orientable genus. Sufficiency was established by the classic work of Ringel and Youngs and is presented in [5]. The set of faces form a twofold triple system of order $n, \operatorname{TTS}(n)$ for short, i.e. a collection of triples having the property that every pair is contained in precisely two triples. In such a triangulation the number of faces around each vertex is $n-1$, and so if $n-1$ is even, i.e. $n \equiv 3$ or $7(\bmod 12)$, it may be possible to colour each face using one of two colours, say black or white, so that no two faces of the same colour are adjacent. We say that the triangulation is (properly) face two-colourable. The set of faces of each colour class then form a Steiner triple system of order $n, \operatorname{STS}(n)$ for short, i.e. a collection of triples having the property that every pair is contained in precisely one triple. We say that the two $\operatorname{STS}(n)$ s are biembedded in the surface. An obvious question therefore is whether for each $n \equiv 3$ or $7(\bmod 12)$, there is a biembedding of some pair of STS $(n)$ s in an orientable surface. The answer is in the affirmative, the case $n \equiv 3(\bmod 12)$ is dealt with in [5], and the case $n \equiv 7(\bmod 12)$ is dealt with in [8].

However, $\operatorname{STS}(n)$ s exist for all $n \equiv 1$ or $3(\bmod 6)$, i.e. $n \equiv 1,3,7$ or $9(\bmod 12)$, [3]. In this paper we are concerned with the case where $n \equiv 1(\bmod 12)$ and consider the question of how close it is possible to biembed a pair of STS $(n)$ s of these orders in an orientable surface. Exactly what we mean by this is now explained. A pseudosurface is the topological space which results when finitely many identifications of finitely many points each are made on a given surface. More precisely, distinct points $\left\{p_{i, j}: i=\right.$ $\left.1,2, \ldots, k, j=1,2, \ldots, m_{i}\right\}$ on a given surface are identified to form points $p_{i}=\left\{p_{i, j}\right.$ : $\left.j=1,2, \ldots m_{i}\right\}, i=1,2, \ldots, k$ called singular points or pinch points. The number $m_{i}$ is the multiplicity of the pinch point. Further, if the rotation about a pinch point consists of cycles of equal length, the pinch point will be called regular. The pseudosurface 
will be called orientable or non-orientable depending on whether the surface from which it is obtained is orientable or non-orientable respectively. In what follows, the pseudosurfaces which we will consider are orientable and contain just one pinch point which is regular and of multiplicity 2, i.e. the rotation about it will consist of two cycles each of length $(n-1) / 2$. Thus, in a sense the pseudosurfaces are as close to being an orientable surface as possible. Euler's formula does not disallow possible biembeddings of pairs of $\operatorname{STS}(n)$ s for $n \equiv 1$ or $9(\bmod 12)$ in these pseudosurfaces, and in this paper we initiate the study of these pseudosurfaces. In particular, we prove that there exists a biembedding in such a pseudosurface of a pair of $\operatorname{STS}(n)$ s for all $n \equiv 13$ or $37(\bmod 72)$. However, first we present some computational results for the smallest value, $n=13$. We will need such a biembedding not only to prove our general result but also to illustrate the idea described in this Introduction.

2. Biembeddings of STS(13)s. Up to isomorphism, there exist precisely two STS(13)s [1]. One of these has a cyclic automorphism and can be constructed on the base set $\mathbb{Z}_{13}$ by the action of the group generated from the mapping $i \mapsto i+1$ (mod 13$)$ on the two starter blocks $\{0,1,4\}$ and $\{0,2,7\}$. Denote this system by $S$. The system also has a further automorphism $\tau: i \mapsto 3 i(\bmod 13)$ of order 3 giving the full automorphism group of order 39 . Without loss of generality we can assume that the point 0 is the pinch point. We then applied all $12 ! / 2^{6} 6 !=10,395$ involutions with no fixed points on the set $\mathbb{Z}_{13} \backslash\{0\}$ in turn to $S$ and tested whether the system obtained biembeds with $S$ in an orientable pseudosurface with 0 as a regular pinch point of multiplicity 2 . There are two involutions which produce such biembeddings, $\sigma=(110)(28)(34)(57)(611)\left(9\right.$ 12) and $\sigma^{\prime}=\left(\begin{array}{ll}1 & 12\end{array}\right)(28)(310)(49)(57)(611)$. The full automorphism group of both the biembeddings is the cyclic group $\mathbb{C}_{6}$, generated respectively by the permutations $g=\sigma \tau=\tau \sigma=\left(\begin{array}{llllll}1 & 4 & 9 & 10 & 3 & 12\end{array}\right)\left(\begin{array}{llllll}2 & 11 & 5 & 8 & 6 & 7\end{array}\right)$ and $g^{\prime}=\sigma^{\prime} \tau=\tau \sigma^{\prime}=\left(\begin{array}{llllll}1 & 10 & 9 & 12 & 3 & 4\end{array}\right)\left(\begin{array}{llllll}2 & 11 & 5 & 8 & 6 & 7\end{array}\right)$. Automorphisms of even order are colour-reversing and those of odd order are colour-preserving. All automorphisms are orientation-preserving. However, the two biembeddings are nonisomorphic and are shown in Tables 1 and 2.

The non-isomorphism of the two biembeddings can be proved by counting the number of Pasch configurations in each TTS(13); there are 112 and 82 Pasch configurations respectively. The biembeddings can also be derived from voltage graphs.

The voltages in the voltage graph shown in Figure 1 are taken in the group $\mathbb{Z}_{3}=\{0,1,2\}$. Therefore, the embedding derived from this voltage graph has a vertex set $\left\{u_{i}^{0}, u_{i}^{1}, u_{i}^{2}: i=0,1,2,3\right\}$. Moreover, the embedding has 20 black triangular faces, 20 white triangular faces and two 6-cycles. The black triangles are $\left\{u_{0}^{i} u_{1}^{i} u_{1}^{i+2}\right\},\left\{u_{0}^{i} u_{0}^{i+1} u_{3}^{i+1}\right\}$, $\left\{u_{0}^{i+1} u_{2}^{i} u_{3}^{i}\right\},\left\{u_{1}^{i} u_{2}^{i+1} u_{3}^{i+2}\right\},\left\{u_{0}^{i} u_{1}^{i+1} u_{2}^{i+1}\right\},\left\{u_{1}^{i} u_{2}^{i+2} u_{3}^{i+1}\right\}, i \in\{0,1,2\} ;\left\{u_{2}^{0} u_{2}^{1} u_{2}^{2}\right\}$ and $\left\{u_{3}^{0} u_{3}^{1} u_{3}^{2}\right\}$, the white triangles are $\left\{u_{0}^{i} u_{2}^{i} u_{3}^{i}\right\},\left\{u_{0}^{i} u_{1}^{i} u_{3}^{i+1}\right\},\left\{u_{0}^{i} u_{1}^{i+1} u_{2}^{i+2}\right\},\left\{u_{0}^{i} u_{1}^{i+2} u_{2}^{i+2}\right\},\left\{u_{1}^{i} u_{2}^{i} u_{2}^{i+2}\right\}$, $\left\{u_{2}^{i} u_{3}^{i+1} u_{3}^{i+2}\right\}, i \in\{0,1,2\} ;\left\{u_{0}^{0} u_{0}^{1} u_{0}^{2}\right\}$ and $\left\{u_{1}^{0} u_{1}^{1} u_{1}^{2}\right\}$ and the 6-cycles are $\left\{u_{0}^{0} u_{2}^{0} u_{0}^{2} u_{2}^{2} u_{0}^{1} u_{2}^{1}\right\}$ and $\left\{u_{1}^{0} u_{3}^{0} u_{1}^{1} u_{3}^{1} u_{1}^{2} u_{3}^{2}\right\}$. Let $u_{0}^{0}=1, u_{0}^{1}=3, u_{0}^{2}=9, u_{1}^{0}=2, u_{1}^{1}=6, u_{1}^{2}=5, u_{2}^{0}=4, u_{2}^{1}=12$, $u_{2}^{2}=10, u_{3}^{0}=7, u_{3}^{1}=8$ and $u_{3}^{2}=11$. Finally, by adding the point 0 to the embedding and connecting it to the other 12 points gives the biembedding of a pair of STS(13)s in an orientable surface with one pinch point with the rotation scheme given in Table 1. A similar approach gives the second biembedding from the voltage graph as shown in Figure 2. 
Table 1. Rotation scheme of biembedding \#1

\begin{tabular}{lcccccccccccc}
\hline $0:$ & $(1$ & 4 & 9 & 10 & 3 & $12)$ & $(2$ & 7 & 6 & 8 & 5 & $11)$ \\
$1:$ & 4 & 0 & 12 & 6 & 10 & 11 & 5 & 2 & 8 & 3 & 9 & 7 \\
$2:$ & 7 & 0 & 11 & 12 & 9 & 4 & 10 & 8 & 1 & 5 & 6 & 3 \\
$3:$ & 12 & 0 & 10 & 5 & 4 & 7 & 2 & 6 & 11 & 9 & 1 & 8 \\
4: & 0 & 1 & 7 & 3 & 5 & 8 & 11 & 6 & 12 & 10 & 2 & 9 \\
$5:$ & 11 & 0 & 8 & 4 & 3 & 10 & 12 & 7 & 9 & 6 & 2 & 1 \\
$6:$ & 8 & 0 & 7 & 10 & 1 & 12 & 4 & 11 & 3 & 2 & 5 & 9 \\
$7:$ & 0 & 2 & 3 & 4 & 1 & 9 & 5 & 12 & 11 & 8 & 10 & 6 \\
$8:$ & 0 & 6 & 9 & 12 & 3 & 1 & 2 & 10 & 7 & 11 & 4 & 5 \\
$9:$ & 10 & 0 & 4 & 2 & 12 & 8 & 6 & 5 & 7 & 1 & 3 & 11 \\
$10:$ & 0 & 9 & 11 & 1 & 6 & 7 & 8 & 2 & 4 & 12 & 5 & 3 \\
$11:$ & 0 & 5 & 1 & 10 & 9 & 3 & 6 & 4 & 8 & 7 & 12 & 2 \\
$12:$ & 0 & 3 & 8 & 9 & 2 & 11 & 7 & 5 & 10 & 4 & 6 & 1 \\
\hline
\end{tabular}

Table 2. Rotation scheme of biembedding \#2

\begin{tabular}{lcccccccccccc}
\hline 0: & $(1$ & 4 & 3 & 12 & 9 & $10)$ & $(7$ & 2 & 11 & 5 & 8 & $6)$ \\
1: & 4 & 0 & 10 & 11 & 12 & 6 & 8 & 3 & 9 & 7 & 5 & 2 \\
2: & 0 & 7 & 9 & 4 & 1 & 5 & 6 & 3 & 8 & 10 & 12 & 11 \\
3: & 12 & 0 & 4 & 7 & 10 & 5 & 11 & 9 & 1 & 8 & 2 & 6 \\
4: & 0 & 1 & 2 & 9 & 8 & 5 & 12 & 10 & 6 & 11 & 7 & 3 \\
5: & 0 & 11 & 3 & 10 & 9 & 6 & 2 & 1 & 7 & 12 & 4 & 8 \\
6: & 0 & 8 & 1 & 12 & 3 & 2 & 5 & 9 & 11 & 4 & 10 & 7 \\
$7:$ & 2 & 0 & 6 & 10 & 3 & 4 & 11 & 8 & 12 & 5 & 1 & 9 \\
8: & 6 & 0 & 5 & 4 & 9 & 12 & 7 & 11 & 10 & 2 & 3 & 1 \\
$9:$ & 10 & 0 & 12 & 8 & 4 & 2 & 7 & 1 & 3 & 11 & 6 & 5 \\
$10:$ & 0 & 9 & 5 & 3 & 7 & 6 & 4 & 12 & 2 & 8 & 11 & 1 \\
$11:$ & 5 & 0 & 2 & 12 & 1 & 10 & 8 & 7 & 4 & 6 & 9 & 3 \\
12: & 0 & 3 & 6 & 1 & 11 & 2 & 10 & 4 & 5 & 7 & 8 & 9 \\
\hline
\end{tabular}

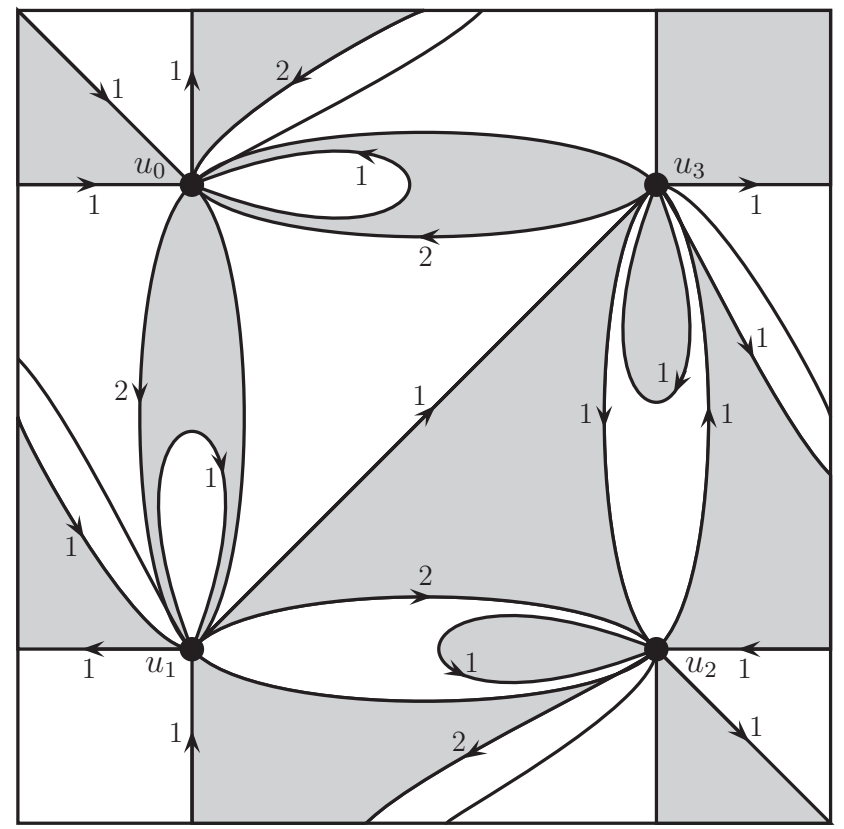

Figure 1. Toroidal embedding of voltage graph of biembedding \#1. 


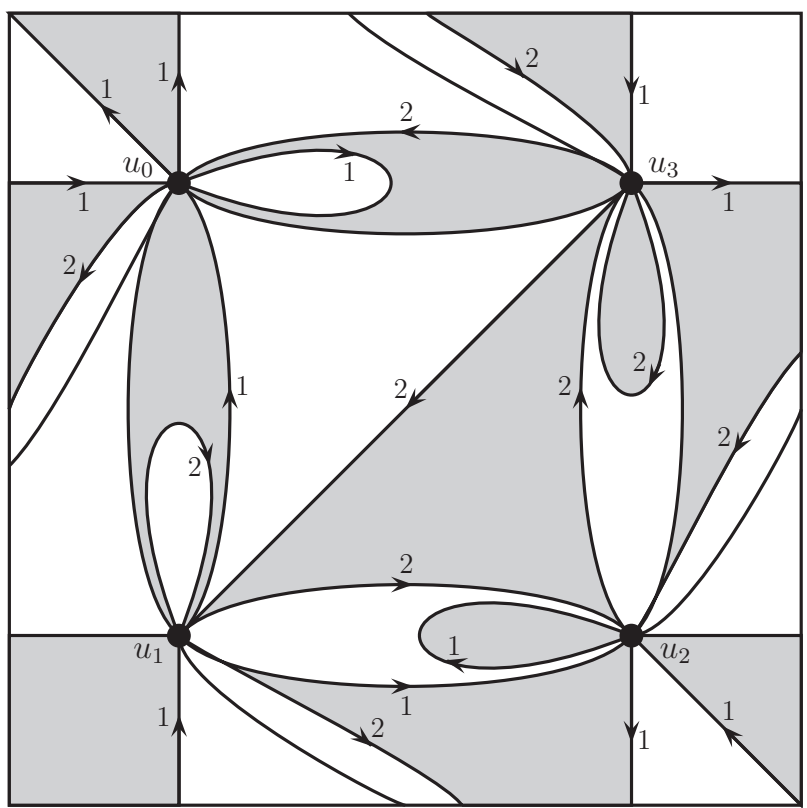

Figure 2. Toroidal embedding of voltage graph of biembedding \#2.

For the other STS(13) we take the representation given in [4], and for sake of completeness list the triples, omitting set brackets for clarity.

$$
\begin{aligned}
& 123,145,167,189,1 a b, 1 c d, 246,257,28 a, 29 c, 2 b d, 348,35 c \\
& 36 d, 37 b, 39 a, 479,4 a d, 4 b c, 56 a, 58 b, 59 d, 68 c, 69 b, 78 d, 7 a c
\end{aligned}
$$

The full automorphism group is the dihedral group $\mathbb{D}_{3}$ of order 6 generated by the permutations $(128)(3 a 9)(4 b c)(5 d 6)$ and $(15)(26)(3 a)(8 d)(b c)$.

The automorphism partitioning is $\{1,2,5,6,8, d\},\{3,9, a\},\{4, b, c\},\{7\}$. Without loss of generality, there are therefore four possibilities for the pinch point, namely $1,3,4$ and 7. We in turn considered each of these by applying the 10,395 involutions without fixed points as in the case of the cyclic STS(13) above. The results are summarized below.

\section{Pinch point 1.}

There are three permutations which give biembeddings. These are $(25)(3 d)(4 c)$ $(6 b)(78)(9 a),(26)(3 c)(4 b)(59)(7 d)(8 a)$ and $(2 c)(39)(4 a)(56)(7 b)(8 d)$. The three biembeddings are non-isomorphic. The TTS(13)s obtained from the biembeddings contain 78, 82 and 98 Pasch configurations respectively.

Pinch point 3.

There are two permutations which give biembeddings. These are $\left(\begin{array}{lll}1 & d\end{array}\right)\left(\begin{array}{ll}2 & 8\end{array}\right)\left(\begin{array}{ll}4 & 6\end{array}\right)$ $(59)(7 c)(a b)$ and $(1 c)(26)(47)(5 d)(8 a)(9 b)$ but the two biembeddings are isomorphic under the permutation $(16)(2 d)(4 c)(58)(9 a)$. The TTS(13) contains 106 Pasch configurations. 
Pinch point 4 .

There are four permutations which give biembeddings. These are $(1 b)(2 c)(37)(56)$ $\left(\begin{array}{ll}8 & d\end{array}\right)(9 a),\left(\begin{array}{ll}1 & b\end{array}\right)(2 \quad 7)(3 \quad c)(5 \quad 8)(6 a)(9 \quad d),\left(\begin{array}{lll}1 & d\end{array}\right)(2 \quad 3)(5 \quad c)(6 \quad 7)(8 \quad 9)\left(\begin{array}{ll}a & b\end{array}\right)$ and $\left(\begin{array}{ll}1 & 2\end{array}\right)(3 \quad 9)(5 c)(6 \quad b)(7 a)(8 d)$. The biembeddings given by permutations \#1 and $\# 4$, and those by permutations \#2 and \#3 are isomorphic under the permutation $(15)(26)(3 a)(8 d)(b c)$. The TTS(13) of the biembedding given by permutations \#1 or \#4 contains 116 Pasch configurations and that given by permutations \#2 or \#3 contains 80 Pasch configurations.

Pinch point 7 .

There are no biembeddings.

The six biembeddings only have the identity and the involution given as automorphisms.

3. General construction. Our main construction is a modification of the product construction described as Construction 4 of [2]. To facilitate a comparison of the steps carried out here with the original proof, we will keep to the notation of [2] as much as possible.

THEOREM 3.1. Suppose that $n \equiv 1(\bmod 12)$ and that $m \equiv 1$ or $3(\bmod 6)$. Then if there exists a biembedding of a pair of Steiner triple systems of order $n$ in an orientable pseudosurface having precisely one regular pinch point of multiplicity 2 , then there exists a biembedding of a pair of Steiner triple systems of order $m(n-1)+1$ in an orientable pseudosurface having precisely one regular pinch point of multiplicity 2.

Proof. We first give a rough outline of the proof. We will begin with taking $m$ copies of a pair of biembedded Steiner triple systems of order $n$ in an orientable surface with one regular pinch point of multiplicity 2, removing the $m$ pinch points together with interiors of incident edges and faces, and 'bridging' the $m$ components in an intricate way to obtain a connected surface with $2 m$ cyclic boundary components. We will continue by capping the $2 m$ 'holes' created in the previous step by a cap consisting of a bordered pseudosurface with one pinch point and $2 m$ cyclic boundary components. The task will be completed by constructing a pair of biembedded STS of order $m(n-1)+1$ out of the partial embedding on the pseudosurface obtained by capping.

Instead of speaking about biembedded STS of order $n$, we will use the equivalent language of face-2-colourable orientable triangular embeddings of $K_{n}$, called simply 2 to-embeddings in [2]. Initially we will assume that $m$ and $(n-1) / 2$ are relatively prime, and we will deal with the general case at the end of the proof.

Let $\eta$ be a 2to-embedding of $K_{n}$ in an (say, clockwise) oriented pseudosurface with a single regular pinch point of multiplicity 2 , with faces properly coloured black and white. Let $z$ be the unique vertex of $K_{n}$ identified with the pinch point. We remove from $\eta$ the vertex $z$, together with all open arcs and open triangular faces originally incident with $z$, obtaining a face 2-coloured triangular embedding $\phi$ of $G=K_{n} \backslash\{z\} \cong K_{n-1}$ in a bordered surface $S$. Observe that $S$ has no pinch points and the two connected boundary components of $S$ are two disjoint cycles $D_{1}$ and $D_{2}$ in $G$, each of length $(n-1) / 2$. Following our outline, for every $i \in Z_{m}$, let $\phi^{i}: G^{i} \rightarrow S^{i}$ be $m$ mutually disjoint copies of the embedding $\phi$ together with the proper 2-colouring of triangular 
faces inherited from $\eta$. In doing so we assume that the natural mapping $f^{i}: G \rightarrow G^{i}$ that endows each vertex of $G$ with the superscript $i$ is a colour-preserving and orientationpreserving isomorphism of the embeddings $\phi$ and $\phi^{i}$.

We continue with describing the 'bridging' procedure. To do so we need to return to the embedding $\phi$ whose description uses no superscripts. Let $\mathcal{T}$ be the set of the total of $t=(n-1)(n-3) / 6$ white triangular faces in $\phi$ and for each $i \in Z_{m}$ let $\mathcal{T}^{i}=f^{i}(\mathcal{T})$ be the corresponding set of all white triangular faces in $\phi^{i}$. Choose a particular triangular face $T$ of $\phi$ with vertex set $\{a, b, c\}$ and assume that the cyclic permutation $(a b c)$ corresponds to the clockwise orientation of the boundary cycle $C$ of $T$. For each such $T$ take a 2to-embedding $\psi_{T}$ of the complete tripartite graph $K_{m, m, m}$ in a closed surface $S_{T}$ disjoint from each $S^{i}$; let $\left\{a_{T}^{i}\right\},\left\{b_{T}^{i}\right\}$ and $\left\{c_{T}^{i}\right\}\left(i \in Z_{m}\right)$ be the three vertex-parts of this $K_{m, m, m}$. Such embeddings are well known, [6, 7]. By Construction 1 of [2], we may select $\psi_{T}$ to have a parallel class of black triangular faces $\left\{a_{T}^{i}, b_{T}^{i}, c_{T}^{i}\right\}$ and we may choose the orientation of $\psi_{T}$ to ensure that it induces the cyclic permutations $\left(a_{T}^{i} c_{T}^{i} b_{T}^{i}\right)$ of the boundary cycles $C_{T}^{i}$ of these faces. Note that we have chosen different cyclic permutations $(a b c)$ on $S$ and $\left(a_{T}^{i} c_{T}^{i} b_{T}^{i}\right)$ on $S_{T}$.

Next, for every $i \in Z_{m}$ we perform the following steps: remove from $\phi^{i}$ the open triangular face $T^{i}=f^{i}(T)$, creating in each $S^{i}$ a new hole with boundary curve $C^{i}=$ $f^{i}(C)$ corresponding to the 3 -cycle $\left(a^{i} b^{i} c^{i}\right)$ in $\phi^{i}$, remove from $\psi_{T}$ the open triangular faces $\left\{a_{T}^{i}, b_{T}^{i}, c_{T}^{i}\right\}$, and identify the closed curve $C^{i}$ in $\phi^{i}$ with the curve $C_{T}^{i}$ in $\psi_{T}$ in such a way that $a^{i} \equiv a_{T}^{i}, b^{i} \equiv b_{T}^{i}$ and $c^{i} \equiv c_{T}^{i}$. Assuming that the embeddings $\psi_{T}$ are mutually disjoint, we apply this procedure successively to each white triangular face $T \in \mathcal{T}$. Let $\hat{S}$ denote the connected triangulated surface $\hat{S}$ with $2 m$ boundary components, obtained this way from the surfaces $S^{i}$. Roughly speaking, $\hat{S}$ is obtained from the surfaces $S^{i}$ by adding $|\mathcal{T}|$ 'bridges', explaining the term 'bridging' used in the earlier informal outline of our construction.

The $2 m$ boundary components of $\hat{S}$ correspond, for $i \in Z_{m}$, to the cycles $D_{1}^{i}=$ $f^{i}\left(D_{1}\right)$ and $D_{2}^{i}=f^{i}\left(D_{2}\right)$ in the graphs $G^{i}$, the images of the cycles $D_{1}$ and $D_{2}$ in $G$. The chosen orientations of $\phi^{i}$ and $\psi_{T}$ induce an orientation of $\hat{S}$ by inheriting the clockwise orientation from $\phi^{i}$ and $\psi_{T}$, and $\hat{S}$ also inherits the proper 2-colouring of triangular faces from these embeddings. Note that there are $t=(n-1)(n-3) / 6$ black triangles in $S$ (and hence in each $S^{i}$ ), and for each of the $t$ white triangles $T$ in $S$ we added, in $\psi_{T}$, another $\left(2 m^{2}-m\right)$ triangles. The total number of triangular faces on $\hat{S}$ is therefore equal to $m t+\left(2 m^{2}-m\right) t=m^{2}(n-1)(n-3) / 3$. For each collection of $\left(2 m^{2}-m\right)$ triangles added, $m^{2}$ are white and $\left(m^{2}-m\right)$ are black; hence, it is easy to check that exactly half of the triangles on $\hat{S}$ are black, as expected.

To proceed, we need an exact description of the graph $H$ triangulating the bordered surface $\hat{S}$. Let $D_{1}=\left(u_{1} u_{2} \ldots u_{(n-1) / 2}\right)$ and $D_{2}=\left(v_{1} v_{2} \ldots v_{(n-1) / 2}\right)$ be the two cycles in $G=K_{n} \backslash\{z\}$ introduced earlier. Since $(n-1) / 2$ is even, every other edge of both $D_{1}$ and $D_{2}$ is incident to a white triangle on $\hat{S}$; let these edges be $u_{2} u_{3}$, $u_{4} u_{5}, \ldots, u_{(n-1) / 2} u_{1}$ and $v_{2} v_{3}, v_{4} v_{5}, \ldots, v_{(n-1) / 2} v_{1}$. It may now be checked that the graph $H$ is obtained as follows. For $1 \leq j \neq j^{\prime} \leq(n-1) / 2$, each vertex $u_{j}$ and $v_{j}$ of $G$ gives rise to $m$ vertices $u_{j}^{i}$ and $v_{j}^{i}$, $(0 \leq i \leq m-1)$, of $H$, and each edge $u_{j} u_{j^{\prime}}$ and $v_{j} v_{j^{\prime}}$ of $G$ incident to a white triangle gives rise to $m^{2}$ edges $u_{j}^{i} u_{j^{\prime}}^{i^{\prime}}$ and $v_{j}^{i} v_{j^{\prime}}^{i^{\prime}}$, $\left(i, i^{\prime} \in Z_{m}\right)$, of $H$. Since each edge of $G$ except for the $(n-1) / 2$ edges $u_{1} u_{2}, u_{3} u_{4}, \ldots$, $u_{(n-3) / 2} u_{(n-1) / 2}$ and $v_{1} v_{2}, v_{3} v_{4}, \ldots, v_{(n-3) / 2} v_{(n-1) / 2}$ is incident to exactly one white triangle, $H$ has $m^{2}(|E(G)|-(n-1) / 2)+m(n-1) / 2=m(n-1)(m(n-3)+1) / 2$ edges. To have further insight into its structure, observe that for each edge $u_{j} u_{j^{\prime}}$ and $v_{j} v_{j^{\prime}}$ of $G \cong K_{n-1}$ 


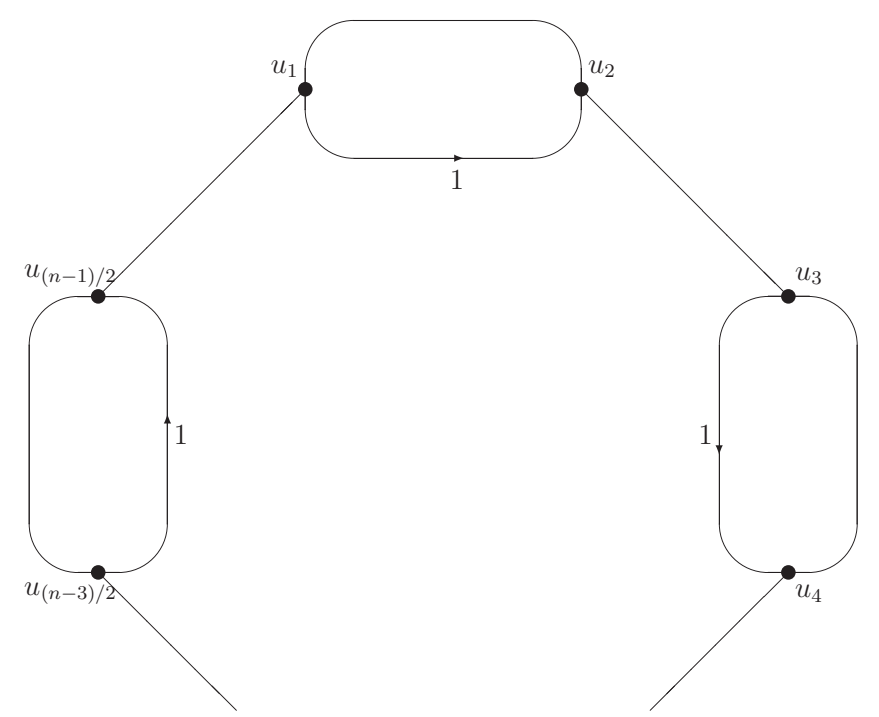

Figure 3. The plane embedding $\mu_{1}$ of the graph $M_{1}$.

(except when $\left\{u_{j}, u_{j^{\prime}}\right\}=\left\{u_{l}, u_{l+1}\right\}$ and $\left\{v_{j}, v_{j^{\prime}}\right\}=\left\{v_{l}, v_{l+1}\right\}, l=1,3,5, \ldots,(n-3) / 2$ ), $H$ contains all edges of the form $u_{j}^{i} u_{j^{\prime}}^{i^{\prime}}$ and $v_{j}^{i} v_{j^{\prime}}^{i^{\prime}}, i, i^{\prime} \in Z_{m}$. But if $\left\{u_{j}, u_{j^{\prime}}\right\}=\left\{u_{l}, u_{l+1}\right\}$ or $\left\{v_{j}, v_{j^{\prime}}\right\}=\left\{v_{l}, v_{l+1}\right\}$ for some $l=1,3, \ldots,(n-3) / 2$ then $H$ contains no edge $u_{j}^{i} u_{j^{\prime}}^{i^{\prime}}$ and $v_{j}^{i} v_{j^{\prime}}^{i^{\prime}}$ with $i \neq i^{\prime}$, although it does contain the edges $u_{j}^{i} u_{j^{\prime}}^{i}$ and $v_{j}^{i} v_{j^{\prime}}^{i}$. Note also that $H$ contains no edges of the form $u_{j}^{i} u_{j}^{i^{\prime}}$ and $v_{j}^{i} v_{j}^{i^{\prime}}$ for any $i, i^{\prime} \in Z_{m}$. It follows that $H$ is isomorphic to $K_{m(n-1)}$ minus $(n-1) / 2$ pairwise disjoint copies of $\left(K_{2 m}\right.$ minus a 1 -factor), one on each of the sets $\left\{u_{l}^{0}, u_{l}^{1}, \ldots, u_{l}^{m-1}, u_{l+1}^{0} u_{l+1}^{1}, \ldots, u_{l+1}^{m-1}\right\}$ and $\left\{v_{l}^{0}, v_{l}^{1}, \ldots, v_{l}^{m-1}, v_{l+1}^{0} v_{l+1}^{1}, \ldots, v_{l+1}^{m-1}\right\}$ with the missing 1 -factor $\left\{u_{l}^{i} u_{l+1}^{i} ; i \in Z_{m}\right\}$ and $\left\{v_{l}^{i} v_{l+1}^{i} ; i \in Z_{m}\right\}$, respectively, for $l=1,3,5, \ldots,(n-3) / 2$.

Let $\omega: H \rightarrow \hat{S}$ be the resulting embedding of $H$ in our surface $\hat{S}$ with $2 m$ boundary components consisting of the images of the cycles $D_{1}$ and $D_{2}$ under the isomorphisms $f^{i}, i \in Z_{m}$. To construct the final 2to-embedding of $K_{m(n-1)+1}$ we build two auxiliary triangulated bordered surfaces $S_{1}^{*}$ and $S_{2}^{*}$ containing $m$ boundary components each, and paste them to $\hat{S}$ so that the $2 m$ holes of $\hat{S}$ will be capped. We will focus on $S_{1}^{*}$ in detail and then explain how $S_{2}^{*}$ is obtained. The surface $S_{1}^{*}$ will be described as a lift of the plane embedding $\mu_{1}$ of the multigraph $M_{1}$ as depicted in Figure 3, with voltages $\alpha$ on directed edges of $M_{1}$ in the group $Z_{m}$ identical with the group from which all our superscripts are taken. Edges with no direction assigned are assumed to carry zero voltage.

The lifted graph $M_{1}^{\alpha}$ has the vertex set $\left\{u_{j}^{i}: 1 \leq j \leq(n-1) / 2, i \in Z_{m}\right\}$. (We are deliberately using the same letters for vertices of $M^{\alpha}$ as for vertices of the graphs $G^{i}$, but assume that these graphs are disjoint; such notation will be of advantage later.) The lifted embedding $\mu_{1}^{\alpha}: M_{1}^{\alpha} \rightarrow R_{1}$ is orientable and has the following face boundaries.

(a) $(n-1) / 4$ faces whose boundaries correspond to cycles of length $2 m$ of the form $\left(u_{2 j-1}^{0} u_{2 j}^{0} u_{2 j-1}^{m-1} u_{2 j}^{m-1} \ldots u_{2 j}^{1}\right)$ for $1 \leq j \leq(n-1) / 4$.

(b) $m$ faces whose boundaries correspond to cycles of length $(n-1) / 2$ of the form $\left(u_{(n-1) / 2}^{i} u_{(n-3) / 2}^{i} \ldots u_{1}^{i}\right)$ for $i \in Z_{m}$. 
(c) One face whose boundary corresponds to a cycle of length $m(n-1) / 2$ of the form $\left(u_{1}^{0} u_{2}^{1} u_{3}^{1} u_{4}^{2} u_{5}^{2} u_{6}^{3} \ldots u_{(n-1) / 2}^{0}\right)$. (Note: This is the only place in this proof where we have used the assumption that $m$ and $(n-1) / 2$ are relatively prime; if this were not the case then a multiplicity of faces with shorter boundary cycles would be obtained.)

We now describe a series of modifications of the embedding $\mu_{1}$. Firstly, we remove all the open faces of type (a) from the surface $R_{1}$, leaving an orientable surface $R_{1}^{o}$ with $(n-1) / 4$ vertex-disjoint boundaries $\left(u_{2 j-1}^{0} u_{2 j}^{0} u_{2 j-1}^{m-1} u_{2 j}^{m-1} \ldots u_{2 j}^{1}\right), 1 \leq j \leq(n-1) / 4$. We cap each of these in turn by taking, for each $j$, a 2 to-embedding of $K_{2 m+1}$ with colour classes black and white on the vertex set $\left\{\infty_{j}, u_{2 j}^{0}, u_{2 j-1}^{0}, u_{2 j}^{1}, u_{2 j-1}^{1}, \ldots, u_{2 j-1}^{m-1}\right\}$, in which the rotation at $\infty_{j}$ is the cycle $\left(u_{2 j}^{0} u_{2 j-1}^{0} u_{2 j}^{1} u_{2 j-1}^{1} \ldots u_{2 j-1}^{m-1}\right)$ and in which the face corresponding to the 3 -cycle $\left(\infty_{j} u_{2 j}^{0} u_{2 j-1}^{0}\right)$ is coloured black. Here also for convenience we are using the same letters for the vertices of our $K_{2 m+1}$ embeddings as for the vertices of $M_{1}^{\alpha}$, but we assume that the corresponding surfaces are disjoint. Secondly, from each embedding of $K_{2 m+1}$ we remove the vertex $\infty_{j}$, all open edges incident with $\infty_{j}$, and all open triangular faces incident with $\infty_{j}$. This results in a face 2-colourable embedding of $K_{2 m}$ in an orientable surface $R_{1 j}$ with a boundary cycle $\left(u_{2 j}^{0} u_{2 j-1}^{0} u_{2 j}^{1} u_{2 j-1}^{1} \ldots u_{2 j-1}^{m-1}\right)$. Thirdly, for every $j$ such that $1 \leq j \leq(n-1) / 4$ we glue the surface $R_{1 j}$ to the surface $R_{1}^{o}$, identifying points carrying the same labels on each of the two surfaces, thereby obtaining an embedding $\mu_{1}^{\prime}: M_{1}^{\prime} \rightarrow R_{1}^{\prime}$ of a graph $M_{1}^{\prime}$ with $m^{2}(n-1) / 2$ edges.

We continue by removing from $R_{1}^{\prime}$ all the open faces of type (b), obtaining thus an orientable surface $S_{1}^{*}$ with $m$ vertex-disjoint boundaries of the form $\left(u_{(n-1) / 2}^{i} u_{(n-3) / 2}^{i} \ldots u_{1}^{i}\right), i \in Z_{m}$.

Let $M_{1}^{*}$ be the graph obtained from $M_{1}^{\prime}$ by adding a new vertex $\infty_{(1)}$ and joining it to each vertex of $M_{1}^{\prime}$ while keeping all other edges in $M_{1}^{\prime}$ unchanged. We construct an embedding $\mu_{1}^{*}: M_{1}^{*} \rightarrow S_{1}^{*}$ from the embedding of $M_{1}^{\prime}$ in $S_{1}^{*}$ by inserting the vertex $\infty_{(1)}$ in the centre of the face $F_{1}$ bounded by the cycle of length $m(n-1) / 2$ and joining this vertex by open arcs within $F_{1}$ to every vertex on the boundary of $F_{1}$ (that is, to every vertex of $\left.M_{1}^{\alpha}\right)$. This gives rise to $m(n-1) / 2$ new triangular faces on $S_{1}^{*}$ bounded, for $1 \leq j \leq(n-1) / 4$, by cycles of the forms $\left(\infty_{(1)} u_{j}^{k} u_{j+1}^{k+1}\right)$ for $j$ odd, and $\left(\infty_{(1)} u_{j}^{k} u_{j+1}^{k}\right)$ for $j$ even. The new triangular faces will be coloured as follows.

The edge $u_{1}^{0} u_{2}^{1}$ lies in a black triangular face of $\mu_{1}^{\prime}$ because $\left(\infty_{1} u_{1}^{0} u_{2}^{1}\right)$ was a white triangular face of the $K_{2 m+1}$ embedding employed in the construction of $\mu_{1}^{\prime}$. We therefore colour white the face of $\mu_{1}^{*}$ bounded by the 3 -cycle $\left(\infty_{(1)} u_{1}^{0} u_{2}^{1}\right)$. It is easy to see that, by an extension of this argument, we must colour white those alternate triangles with boundary cycles $\left(\infty_{(1)} u_{j}^{k} u_{j+1}^{k+1}\right)$ for $j$ odd. The remaining alternate triangles, those with boundary cycles of the form $\left(\infty_{(1)} u_{j}^{k} u_{j+1}^{k}\right)$ for $j$ even, do not share an edge with any existing triangular face of $\mu_{1}^{\prime}$ and these are coloured black.

By this process, the triangular faces of $\mu_{1}^{*}$ are properly 2-coloured, and the number of such faces is

$$
\frac{(n-1)}{4} \frac{2 m(2 m-2)}{3}+\frac{m(n-1)}{2}=\frac{m(2 m+1)(n-1)}{6},
$$

where the terms $(n-1) / 4,2 m(2 m-2) / 3$ and $m(n-1) / 2$ on the left represent the number of faces of type (a) in $R_{1}$, the number of triangles in the added $K_{2 m}$ and the number of triangles added by inserting the vertex $\infty_{(1)}$ respectively. Note that exactly half of these faces are coloured black. 
The next step is to construct an embedding $\mu_{2}^{*}$ of a graph $M_{2}^{*}$ on a surface $S_{2}^{*}$ with an extra vertex $\infty_{(2)}$, which is done in exactly the same way as described above for $\mu_{1}^{*}$ by replacing all occurrences of $u$ with $v$ and keeping all subscripts and superscripts unchanged. The description would thus start from an embedding $\mu_{2}$ of a graph $M_{2}$ with vertices $v_{1}, v_{2}, \ldots, v_{(n-1) / 2}$ corresponding to Figure 3 and continue through the intermediate graphs, surfaces and embeddings $M_{2}^{\alpha}, R_{2}, \mu_{2}^{\alpha}, R_{2}^{o}, M_{2}^{\prime}, R_{2}^{\prime}, \mu_{2}^{\prime}$ to $M_{2}^{*}$, $S_{2}^{*}$ and $\mu_{2}^{*}$ as indicated. The embedding $\mu_{2}^{*}$ will, of course, have the same number of triangles as given by (1), half of which will be black.

We are ready for the final steps. Our method of constructing the orientable surface $\hat{S}$ from the earlier part of the proof guarantees that a chosen orientation of $\hat{S}$ induces consistent orientations of the boundary cycles of the $2 m$ holes of $\hat{S}$. We may assume that the orientation induces the cyclic ordering of the cycles $D_{1}^{i}$ and $D_{2}^{i}$ in the form that was used before, namely $D_{1}^{i}=f^{i}\left(D_{1}\right)=\left(u_{1}^{i} u_{2}^{i} \ldots u_{(n-1) / 2}^{i}\right)$ and $D_{2}^{i}=f^{i}\left(D_{2}\right)=\left(v_{1}^{i} v_{2}^{i} \ldots v_{(n-1) / 2}^{i}\right), i \in Z_{m}$. The bordered surfaces $S_{1}^{*}$ and $S_{2}^{*}$ have $m$ holes each. Our construction again implies that an orientation of $S_{1}^{*}$ and $S_{2}^{*}$ can be chosen so that the boundary cycles are oriented in the form $D_{1}^{* i}=\left(u_{(n-1) / 2}^{i} \ldots u_{2}^{i} u_{1}^{i}\right)$ and $D_{2}^{* i}=\left(v_{(n-1) / 2}^{i} \ldots v_{2}^{i} v_{1}^{i}\right), i \in Z_{m}$. It remains to do the obvious, namely for each $i$ to paste together the boundary cycles $D_{1}^{i}$ and $D_{1}^{* i}$ in such a way that the corresponding vertices $u_{j}^{i}$ get identified, and glue the boundary cycles $D_{2}^{i}$ and $D_{2}^{* i}$ so that the corresponding vertices $v_{j}^{i}$ will be identified. At last, we identify the vertex $\infty_{(1)}$ with $\infty_{(2)}$, creating one regular pinch point of multiplicity 2 . The final result is an orientable pseudosurface $\bar{S}$ with a single pinch point, regular of multiplicity 2 , and a triangular embedding $\sigma: K \rightarrow \bar{S}$ of some graph $K$. We claim that $K \cong K_{m(n-1)+1}$ and that the triangulation is face 2-colourable.

Obviously, $|V(K)|=m(n-1)+1$. A straightforward edge count shows that

$$
\begin{aligned}
|E(K)| & =|E(H)|+\left|E\left(M_{1}^{*}\right)\right|+\left|E\left(M_{2}^{*}\right)\right|-m\left|E\left(D_{1}\right)\right|-m\left|E\left(D_{2}\right)\right| \\
& =\frac{m(n-1)(m(n-3)+1)}{2}+(n-1)\left(m^{2}+m\right)-m(n-1) \\
& =\frac{m(n-1)(m(n-1)+1)}{2}=\left|E\left(K_{m(n-1)+1}\right)\right| .
\end{aligned}
$$

It is easy to check that, except for edges incident with the vertex obtained by identification of $\infty_{(1)}$ with $\infty_{(2)}$ and edges contained in the $2 m$ cycles $D_{1}^{* i}$ and $D_{2}^{* i}$ of length $(n-1) / 2$, the graph $M_{1}^{*} \cup M_{2}^{*}$ contains exactly those edges which are missing in $H$. This shows that there are no repeated edges or loops in $K$, and thus $K \cong K_{m(n-1)+1}$. As regards the face 2-colouring, we just have to see what happens along the identified cycles $D_{1}^{i}$ and $D_{1}^{* i}$, and $D_{2}^{i}$ and $D_{2}^{* i}$, since the triangulations of $\hat{S}, S_{1}^{*}$ and $S_{2}^{*}$ have been face 2-coloured. But according to the construction, if $l=1,3,5, \ldots,(n-3) / 2$, a triangular face on $\hat{S}$ that contains the edge $u_{l}^{i} u_{l+1}^{i}$ is black, while the face on $S_{1}^{*}$ containing this edge is white because the embeddings of $K_{2 m+1}$ employed had the faces with boundary cycles $\left(\infty_{j} u_{2 j}^{i} u_{2 j-1}^{i}\right)$ coloured black. This also applies to the way the embeddings $\hat{S}$ and $S_{2}^{*}$ meet.

To finish the proof it remains to deal with the case when $m$ and $(n-1) / 2$ are not relatively prime. To do so, we return to Figure 3 and generalise the construction. Namely, it turns out that the voltages shown in Figure 3 as 1 may be replaced respectively by voltages $x_{1}, x_{2}, \ldots, x_{(n-1) / 4} \in Z_{m}$, provided that 
(d) each $x_{i}$ is relatively prime to $m$, and

(e) $\sum_{i=1}^{(n-1) / 4} x_{i}$ is relatively prime to $m$.

Condition (d) ensures that the embedding $\mu_{1}^{\alpha}$ will have $(n-1) / 4$ faces with boundary cycles of length $2 m$ on each of the sets of points of the form $\left\{u_{2 j-1}^{0}, u_{2 j}^{0}, u_{2 j-1}^{1}, u_{2 j}^{1}, \ldots, u_{2 j-1}^{m-1}, u_{2 j}^{m-1}\right\}$, while condition (e) ensures that $\mu_{1}^{\alpha}$ has a single face with boundary cycle of length $m(n-1) / 2$. In effect, condition (e) replaces the condition that $m$ and $(n-1) / 2$ should be relatively prime. Of course, a similar conclusion applies to the embedding $\mu_{2}^{\alpha}$. It is easy to see that there are numerous ways to select the voltages so that $x_{j} \in\{+1,-1\}, 1 \leq j \leq(n-1) / 4$, with $\sum_{i=1}^{(n-1) / 4} x_{i} \in\{1,2\}$, which is relatively prime to $m$ since $m$ is odd. The subsequent steps in the proof then proceed as before with the obvious changes. We leave the verification of the details to the reader.

COROLlary 3.1.1. For all $n \equiv 13$ or 37 ( $\bmod 72)$, there exists a biembedding of a pair of Steiner triple systems of order $n$ in an orientable pseudosurface having precisely one regular pinch point of multiplicity 2.

Proof. Put $n=13$ in the above theorem and use one of the biembeddings given in Section 2.

REMARK. The existence of such a biembedding of a pair of STS(25)s would extend the existence spectrum to include all $n \equiv 25$ or $73(\bmod 144)$, i.e. in arithmetic set density terms from $1 / 3$ to $1 / 2$ in the set of all $n \equiv 1(\bmod 12)$. We have tried to construct such a biembedding but have been unsuccessful.

\section{REFERENCES}

1. V. De Pasquale, Sui sistemi ternari di 13 elementi, Rend. R. Ist. Lombardo Sci. Lett. 32 (1899), 213-221.

2. M. J. Grannell, T. S. Griggs and J. Širáň, Recursive constructions for triangulations, $J$. Graph. Theory 39(2) (2002), 87-107.

3. T. P. Kirkman, On a problem in combinations, Cambridge Dublin Math. J. 2 (1847), $191-204$

4. R. A. Mathon, K. T. Phelps and A. Rosa, Small Steiner triple systems and their properties, Ars Combin. 15 (1983), 3-110.

5. G. Ringel, Map color theorem (Springer-Verlag, New York, NY, 1974).

6. G. Ringel and J. W. T. Youngs, Das Geschlecht des vollständige dreifarben Graphen, Comment. Math. Helv. 45 (1970), 152-158.

7. S. Stahl and A. T. White, Genus embeddings for some complete tripartite graphs, Discrete Math. 14 (1976), 279-296.

8. J. W. T. Youngs, The mystery of the Heawood conjecture, in Graph theory and its applications (Academic Press, New York, NY, 1970), 17-50. 